\title{
Contextuality and Nonlocality in Decaying Multipartite Systems
}

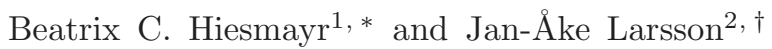 \\ ${ }^{1}$ Faculty of Physics, University of Vienna, Boltzmanngasse 5, 1090 Vienna, Austria \\ ${ }^{2}$ Department of Electrical Engineering, Linköping University, 58183 Linköping, Sweden
}

\begin{abstract}
Everyday experience supports the existence of physical properties independent of observation in strong contrast to the predictions of quantum theory. In particular, existence of physical properties that are independent of the measurement context is prohibited for certain quantum systems. This property is known as contextuality. This paper studies whether the process of decay in space-time generally destroys the ability of revealing contextuality. We find that in the most general situation the decay property does not diminish this ability. However, applying certain constraints due to the space-time structure either on the time evolution of the decaying system or on the measurement procedure, the criteria revealing contextuality become inherently dependent on the decay property or an impossibility. In particular, we derive how the context-revealing setup known as Bell's nonlocality tests changes for decaying quantum systems. Our findings illustrate the interdependence between hidden and local hidden parameter theories and the role of time.
\end{abstract}

Introduction. - The notion of (non)-contextuality has its origins in logic of non-simultaneously decidable propositions [1] and has been extensively studied, in particular with respect to the question of the existence of hidden parameters [2] and in terms of applications such as being the key property for a computation speed up in quantum algorithms [3 $[-5]$. A theory is said to be non-contextual if every random variable only depends on the choice of the measurement but not on the choice of other compatible measurements that are co-measured, its measurement context. If this independence condition does not hold, we call it contextual. This property can be tested through criteria designed such that they distinguish these two cases given the conditions, e.g. [2, 6] 8]. Another way to formulate this is to view measurements in groups that are compatible to each other, contexts, as having outcomes that are jointly distributed within each context but stochastically unrelated between contexts. In quantum mechanics different contexts correspond to different mutually incompatible conditions, so no stochastic relation is present. The question is whether a joint distribution on the full set of observables exists, then providing a non-contextual model, or if it does not exist, so that the system can be said to be contextual.

This paper considers decaying quantum systems and asks whether the process of decay diminishes or destroys the contextual feature present at a certain time point. In particular, we will consider the question whether for a set of measurements, the impossibility of pre-determined outcomes holds for all times if it holds for a certain time in the past (or future). This is a non-trivial question since decaying systems live in Hilbert spaces that have to be separated in a "surviving part" and a "decaying part", where only the surviving part is available for the intended measurements. In particular, the all-important choice of context is only possible for the surviving part.

The paper is organized as follows. First, we stress that there are two different types of dichotomic measurements for decaying (multipartite) systems. We then show that in the joint-particle measurement scenario (defined below) every criterion revealing contextuality can be turned into a criterion that is violated (revealing contextuality) for all times if it is violated at a time point in the past (or future). This proves that the property of contextuality, the impossibility to pre-assign results to a measurement, persists in time, i.e., is unaffected by the decay property. In the case of single-particle measurements (defined below), which are the most common experimental situations, we show that the conditions of compatibility are more involved. Last but not least we elaborate how the specific contextuality test known as "Bell's nonlocality" leads to Bell inequalities for decaying systems. This in particular illuminates how dynamical nonlocality differs with respect to stable systems.

Two distinct dichotomic measurements on a multipartite decaying system. - A decaying system has a natural separation into a "surviving part" and into a "decaying part" whose Hilbert spaces are disjoint. The crucial point is that any experimental setup only has access to the surviving part. Consequently, there exists two dichotomic inequivalent information complete questions that can be raised to an $n$-partite decaying system:

(i) Joint-particle measurements: Is the decaying system in the state $|\psi\rangle=\sum_{i=1}^{d_{1} \cdot d_{2} \cdots \cdot d_{n}} \alpha_{i}\left|e^{(i)}\right\rangle$ at time $t_{1}, t_{2}, \ldots, t_{n}$ or not?

(ii) Single-particle measurements: Is the decaying system in the state $\left|\phi_{1}\right\rangle=\sum_{i=1}^{d_{1}} \alpha_{i}\left|f_{1}^{(i)}\right\rangle$ for particle 1 at time $t_{1}$ or not, in the state $\left|\phi_{2}\right\rangle=\sum_{i=1}^{d_{2}} \beta_{i}\left|f_{2}^{(i)}\right\rangle$ at time $t_{2}$ for particle 2 or not, ..., and in the state $\left|\phi_{n}\right\rangle=\sum_{n=1}^{d_{2}} \gamma_{i}\left|f_{n}^{(i)}\right\rangle$ at time $t_{n}$ for particle $n$ or not?

Here we have assumed that the decaying systems consist of $n$ particles $(n=1,2, \ldots)$ each described by $d_{n}$ degrees of freedom. The vectors $\left|e^{i}\right\rangle,\left|f_{j}^{i}\right\rangle$ form an orthogonal basis of the surviving part of the Hilbert space, 


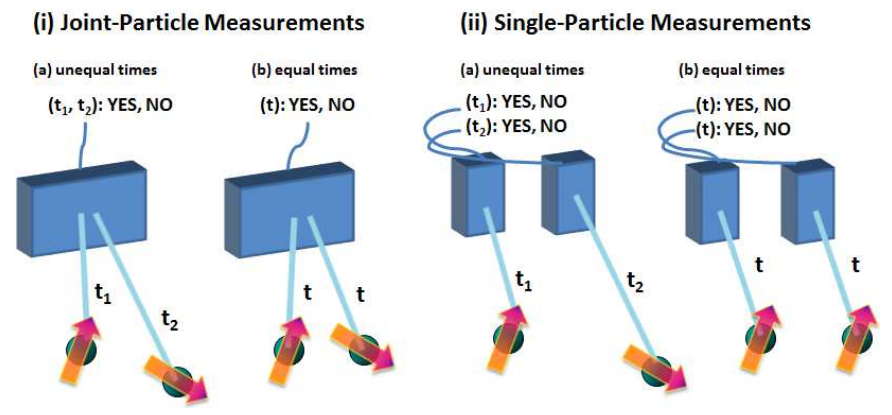

FIG. 1: Schematic view of the two dichotomic questions for a two particle scenario. Note that the two particles can be in a separable or entangled state.

respectively. These two conceptually different measurement procedures and their two different cases (equal and unequal times) are illustrated in Fig. 1]

Time evolution. - Since the decay is a Markov process we can model the system as an open quantum system (for applications see e.g. Refs. [9, 10]). As shown in Ref. [11] any decaying multipartite system can be modeled by a Hamiltonian $H$ covering the surviving part $s$ and a Lindblad operator $L$ connecting the $s$ part with the decaying part $d$, i.e.

$$
\mathcal{H}=|s\rangle\langle s| \otimes H \quad \text { and } \quad \mathcal{L}=|d\rangle\langle s| \otimes L,
$$

which satisfies the Lindblad master equation [12, 13] $(\hbar \equiv$ 1)

$$
\frac{\mathrm{d} \rho}{\mathrm{d} t}=-i[\mathcal{H}, \rho]-\frac{1}{2} \sum\left(\mathcal{L}_{i}^{\dagger} \mathcal{L}_{i} \rho+\rho \mathcal{L}_{i}^{\dagger} \mathcal{L}_{i}-2 \mathcal{L}_{i} \rho \mathcal{L}_{i}^{\dagger}\right)
$$

where $\rho$ is the state of the decaying system and is divided into a "surviving" and a "decaying" part: $\rho=|s\rangle\langle s| \otimes$ $\rho_{s}+|d\rangle\langle s| \otimes \rho_{d}$. Obviously, the decaying part has to be determined by the time evolution of the surviving part, i.e.

$$
\rho_{d}(t)=L \int_{0}^{t} \rho_{s}\left(t^{\prime}\right) d t^{\prime} L^{\dagger}
$$

and the decay rate $\Gamma$ is given by $L^{\dagger} L=\Gamma$. The solution $\rho(t)=|s\rangle\left\langle s\left|\otimes \rho_{s}(t)+\right| d\right\rangle\langle s| \otimes \rho_{d}(t)$ of this differential equations can be derived for any number of particles and is referred to as a "joint-particle" time evolution.

To obtain a "single-particle" time evolution of $n$ particles we have to exploit the usual tensor product structure for the Hamiltonian and the generators of the decay

$$
\begin{aligned}
& H_{\text {single-particle }}= \\
& \quad H \otimes \mathbb{1}^{\otimes n-1}+\mathbb{1} \otimes H \otimes \mathbb{1}^{\otimes n-2}+\cdots+\mathbb{1}^{\otimes n-1} \otimes H \\
& L_{\text {single-particle }}= \\
& \quad L \otimes \mathbb{1}^{\otimes n-1}+\mathbb{1} \otimes L \otimes \mathbb{1}^{\otimes n-2}+\cdots+\mathbb{1}^{\otimes n-1} \otimes L .
\end{aligned}
$$

Note that in this case the total state is divided for two particles into four subspaces, surviving-surviving (ss), surviving-decaying (sd), decaying-surviving (ds) and decaying-decaying (dd) and defined for two different times. Explicit solutions for both cases are discussed later.

Revealing Contextuality in Decaying Systems and as given by Space-Time structure. - We start by exploiting the state-dependent Klyachko-Can-BinicioğluShumovsky-inequality [6] that works for any system of dimension three or larger. It is given by

$$
\begin{aligned}
I_{K C B S}= & \operatorname{Tr}\left(O_{1} O_{2} \rho\right)+\operatorname{Tr}\left(O_{2} O_{3} \rho\right)+\operatorname{Tr}\left(O_{3} O_{4} \rho\right) \\
& +\operatorname{Tr}\left(O_{4} O_{5} \rho\right)+\operatorname{Tr}\left(O_{5} O_{1} \rho\right) \geq-3,
\end{aligned}
$$

where each pair of observables has to be compatible (which means for quantum mechanics that the observables are orthogonal, i.e. $\left.\operatorname{Tr} O_{i} O_{\bmod (i+1,5)}=0\right)$. For an optimal choice of quantum observables with respect to some given pure state $\rho$ it is known that the quantum bound $5-4 \sqrt{5} \approx-3.944$ can be reached which, consequently, reveals the contextual feature of quantum mechanics. Since the operators $O_{i}$ do not need to have a tensor-product structure they generally correspond to "joint-particle" measurements, type $(i)$, and the relevant time evolution is a joint-particle time evolution. Let us assign the numbers +1 to a YES outcome and -1 to a NO outcome, obviously the physics does not depend on that choice (we will exploit this fact later). Any expectation value can be rewritten to only depend on the surviving part through

$$
\begin{aligned}
\operatorname{Tr} O_{i} \rho & =\operatorname{Tr}\left(2 \mathcal{P}_{i}-\mathbb{1}\right) \rho=\operatorname{Tr}\left(2 P_{i}-\mathbb{1}\right) \rho_{s}-\operatorname{Tr} \rho_{d} \\
& =\operatorname{Tr}\left(2 P_{i}-\mathbb{1}\right) \rho_{s}-\left(1-\operatorname{Tr} \rho_{s}\right),
\end{aligned}
$$

where $\mathcal{P}_{i}$ is a projector on the full space and $P_{i}$ the corresponding projector onto the surviving part (note that no projection onto the decaying part is possible). If we assign instead the numbers -1 to a YES outcome and +1 to a NO outcome, we obtain an overall minus sign, but if we assign this relabelling to the projector $P_{i}$ only onto the decaying part, we obtain a relative sign change. This situation corresponds to two physical distinct questions that are identical for stable systems, i.e., (here we assume for simplicity that all particles are measured jointly at the same time instance)

(A) Is the system in the state $\left|\psi_{i}\right\rangle$ at time $t$ or not?: $\operatorname{Tr} O_{i} \rho(t)=\operatorname{Tr}\left(2 P_{i}-\mathbb{1}\right) \rho_{s}(t)-\left(1-\operatorname{Tr} \rho_{s}(t)\right)$

(B) Is the system not in the state $\left|\psi_{i}^{\perp}\right\rangle$ with $\left\langle\psi \mid \psi^{\perp}\right\rangle=0$ at time $t$ or is it?:

$$
\operatorname{Tr} \bar{O}_{i} \rho(t)=\operatorname{Tr}\left(2 P_{i}-\mathbb{1}\right) \rho_{s}(t)+\left(1-\operatorname{Tr} \rho_{s}(t)\right)
$$

The first question outputs +1 if the system is in the state $\left|\psi_{i}\right\rangle$, while the second question outputs +1 if the system is in the state $\left|\psi_{i}\right\rangle$ or if it has decayed. In a measurement of $O_{i} O_{j}$ there are now two possibilities depending on whether we choose the same or different assignments 
of +1 and -1 to the measurement outcomes, i.e.:

$$
\operatorname{Tr} O_{1} O_{2} \rho=\operatorname{Tr}\left(2 P_{1}-\mathbb{1}\right)\left(2 P_{2}-\mathbb{1}\right) \rho_{s} \pm\left(1-\operatorname{Tr} \rho_{s}\right)
$$

Inserting these expectation values into $I_{K C B S}$ we obtain an inequality for decaying subsystems that reads

$$
\begin{aligned}
I_{K C B S}^{\text {decay }}(t)= & \operatorname{Tr}\left(\left(2 P_{1}-\mathbb{1}\right)\left(2 P_{2}-\mathbb{1}\right) \rho_{s}(t)\right) \\
& +\operatorname{Tr}\left(\left(2 P_{2}-\mathbb{1}\right)\left(2 P_{3}-\mathbb{1}\right) \rho_{s}(t)\right) \\
& +\operatorname{Tr}\left(\left(2 P_{3}-\mathbb{1}\right)\left(2 P_{4}-\mathbb{1}\right) \rho_{s}(t)\right) \\
& +\operatorname{Tr}\left(\left(2 P_{4}-\mathbb{1}\right)\left(2 P_{5}-\mathbb{1}\right) \rho_{s}(t)\right) \\
& +\operatorname{Tr}\left(\left(2 P_{5}-\mathbb{1}\right)\left(2 P_{1}-\mathbb{1}\right) \rho_{s}(t)\right) \\
& +c \cdot\left(1-\operatorname{Tr} \rho_{s}(t)\right) \geq-3 .
\end{aligned}
$$

Due to the freedom of assigning +1 and -1 to the measurement outcomes one can control the additional term $c \cdot\left(1-\operatorname{Tr} \rho_{s}\right)$. The optimum is reached by choosing alternating assignments of -1 and +1 to the event of finding that the system has decayed, resulting in $c_{\mathrm{opt}}=-3$. Since $\rho_{s}$ vanishes with increasing time $t$ the inequality $I_{K C B S}^{\text {decay }}(t \longrightarrow \infty)$ approaches the classical bound -3 from below. Consequently, we have shown that if a decaying system violates this criterion at a given point in time, the violation decreases as time goes on but will remain for all times, thus the contextual feature remains. Note that this result holds only for joint-particle measurements and corresponding joint-particle time evolutions as we will discuss later in detail.

Let us consider another inequality revealing contextuality, the well known Mermin-Peres square [7, 8], which is known to be state-independent

$$
\begin{aligned}
I_{M P}= & \operatorname{Tr}\left\{\left(A_{11} A_{12} A_{13}+A_{21} A_{22} A_{23}+A_{31} A_{32} A_{33}\right.\right. \\
& \left.\left.+A_{11} A_{21} A_{31}+A_{21} A_{22} A_{23}-A_{31} A_{32} A_{33}\right) \rho\right\} \leq 6
\end{aligned}
$$

with

$$
(A)_{i j}=\left(\begin{array}{ccc}
\sigma_{x} \otimes \mathbb{1} & \mathbb{1} \otimes \sigma_{z} & \sigma_{x} \otimes \sigma_{z} \\
\mathbb{1} \otimes \sigma_{x} & \sigma_{z} \otimes \mathbb{1} & \sigma_{z} \otimes \sigma_{x} \\
\sigma_{x} \otimes \sigma_{x} & \sigma_{z} \otimes \sigma_{z} & \sigma_{y} \otimes \sigma_{y}
\end{array}\right)_{i j} .
$$

It involves the product of three operators (being measured jointly!) and that all products compute. For decaying quantum systems we obtain

$$
\begin{aligned}
\operatorname{Tr} O_{1} O_{2} O_{3} \rho= & \pm \operatorname{Tr}\left(2 P_{1}-\mathbb{1}\right)\left(2 P_{2}-\mathbb{1}\right)\left(2 P_{3}-\mathbb{1}\right) \rho_{s}(t) \\
& -\left(1-\operatorname{Tr} \rho_{s}(t)\right),
\end{aligned}
$$

where we obtained again a relative sign depending on our assignment of +1 or -1 to a "YES" event. Thus the Mermin-Peres version for decaying systems (for both sign choices) becomes

$I_{M P}^{\text {decay }}=6 \operatorname{Tr} \rho_{s}(t)+4\left(1-\operatorname{Tr} \rho_{s}(t)\right)=2 \operatorname{Tr} \rho_{s}(t)+4 \leq 4$,

which is obviously violated for any initial state and for all times.

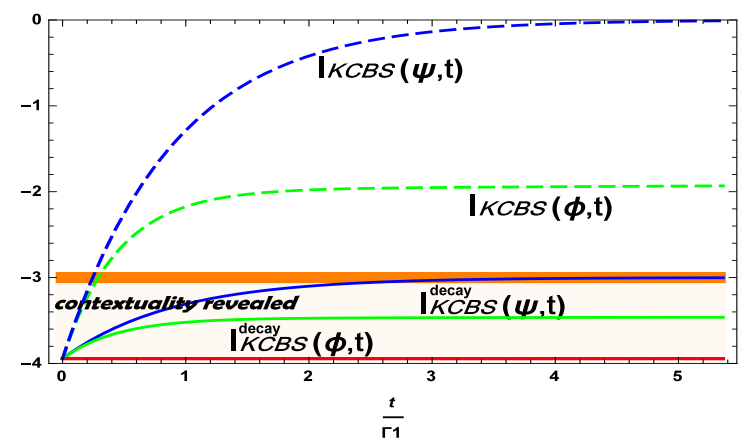

FIG. 2: The curves show the KCBS-inequality optimized over all five observables at each time point for entangled neutral K-meson pairs (in units of time life of the shortest decay rate). The blue curves correspond to initial Bell states $\left|\psi^{ \pm}\right\rangle \equiv \psi$ and the green curves for $\left|\phi^{ \pm}\right\rangle \equiv \phi$ given in the basis of the eigenstates of the Hamiltonian (the mass-eigenstates). The dashed curves are the results of the unoptimized version, Ineq. (5), and the bold curves of the optimized version, Ineq. (8). For longer time scales also $I_{\mathrm{KCBS}}^{\text {decay }}(\phi, t)$ approaches from above the classical bound -3 .

Straightforwardly, one can also optimize the corresponding contextuality criteria for more than two particles, e.g. the state independent criterion for three qubit systems introduced in Ref. [14] becomes

$$
I_{3 \text { particles }}^{\text {decay }}=3+2 \operatorname{Tr} \rho_{s s s}+2 \operatorname{Tr} \rho_{s d s}+2 \operatorname{Tr} \rho_{d s d} \leq 3 .
$$

Again in the limit of infinite time, we approach the bound from above showing that if contextuality can be witnessed by this inequality for a certain time instance, then it holds for all times.

Refining The Contexts By The Space-TimeStructure. - The simplest decaying quantum system is a two-state system (qubit). The solution of the Lindblad equation (2) in terms of Kraus operators $Q_{i}$ and assuming two decays constants $\Gamma_{1,2}$ and two energies $E_{1,2}$ is given by

$$
\rho(t)=\sum_{i=s}^{d} Q_{i}(t) \rho_{s} Q_{i}^{\dagger}(t)
$$

with $Q_{s}(t)=|s\rangle\left\langle s\left|\otimes K_{s}(t), \quad Q_{d}(t)=\right| d\right\rangle\langle s| \otimes$ $K_{d}(t), K_{s}(t)=\operatorname{diag}\left\{e^{-\frac{\Gamma_{1}+i\left(E_{2}-E_{1}\right)}{2} t}, e^{-\frac{\Gamma_{2}-i\left(E_{2}-E_{1}\right)}{2} t}\right\}$ and $K_{d}(t)=\operatorname{diag}\left\{\sqrt{1-e^{-\Gamma_{1} t}}, \sqrt{1-e^{-\Gamma_{2} t}}\right\}$. Obviously both discussed criteria for contextuality can not be violated since at least a three dimensional system for the KCBScriterion or a four dimensional system for the MerminPeres-criterion is required. Therefore we proceed to bipartite identical two-state systems. The joint-particle time evolution in terms of Kraus operators is derived to

$$
\rho(t)=\sum_{i=1}^{2} Q_{i}(t) \rho_{s s} Q_{i}^{\dagger}(t)
$$


with $Q_{1}(t)=|s\rangle\left\langle s\left|\otimes K_{s}(t) \otimes K_{s}(t), Q_{2}(t)=\right| d\right\rangle\langle s| \otimes$ $K_{d d}(t)$ and

$$
\begin{aligned}
K_{d d}(t)=\operatorname{diag}\left\{\sqrt{1-e^{-2 \Gamma_{1} t}}, \sqrt{1-e^{-\left(\Gamma_{1}+\Gamma_{2}\right) t}},\right. \\
\left.\sqrt{1-e^{-\left(\Gamma_{1}+\Gamma_{2}\right) t}}, \sqrt{1-e^{-2 \Gamma_{2} t}}\right\} .
\end{aligned}
$$

Note that in the decay-decay (dd) part the tensorproduct structure in the time parameter is lost.

We can now use the above two criteria for contextuality, KCBS and Mermin-Peres. Fig. 2 shows the result for the flavor-oscillating and decaying K-mesons system for the $K C B S$ criterion $\left(E_{1}-E_{2} \equiv \Delta m=m_{2}-m_{1}=\right.$ $3.5 \cdot 10^{-12} \mathrm{MeV}$ and $\left.\Gamma_{1} \approx 2 \Delta m \approx 600 \Gamma_{2}\right)$. Let us remark that the behaviour of the violation depends strongly on the initial Bell state (symmetric or antisysmmetric) showing an additional state dependence due to the decay property. Initial entangled states for pairs of K-mesons can be produced [15, 16], however, it is not clear how joint-particle measurements may be technically realized. A suitable system for the application of the MerminPeres criterion are spin entangled hyperon-antihyperon systems which also decay via weak interactions but have half-integer spins as discussed in Ref. 17].

Typically in decaying bipartite systems one assumes independent time evolutions for the individual particles. The solution of the Lindblad equation (2) has then to be separated into the four parts $(s s),(s d),(d s)$ and $d d$, i.e. we obtain a state conditioned to the two time choices $t_{l}, t_{r}$ ( $l \ldots$ left,$r \ldots$ right $)$

$$
\rho\left(t_{l}, t_{r}\right) \equiv \sum_{i, j=s}^{d} Q_{i j}\left(t_{l}, t_{r}\right) \rho_{s s} Q_{i j}^{\dagger}\left(t_{l}, t_{r}\right)
$$

with $Q_{i j}\left(t_{l}, t_{r}\right)=|i j\rangle\langle s s| \otimes K_{i}\left(t_{l}\right) \otimes K_{j}\left(t_{r}\right)$. Consequently, the expectation value of two jointly measured observables becomes

$$
\begin{aligned}
\operatorname{Tr}\left\{O_{1} O_{2} \rho\right\}= & \operatorname{Tr}\left\{\left(2 \mathcal{P}_{1}-\mathbb{1}\right)\left(2 \mathcal{P}_{2}-\mathbb{1}\right) \rho\left(t_{l}, t_{r}\right)\right\} \\
= & \sum_{j, k=s}^{d} \operatorname{Tr}\left\{\left(2\left(P_{1}\right)^{j k}-\mathbb{1}\right)\left(2\left(P_{2}\right)^{j k}-\mathbb{1}\right)\right. \\
& \left.Q_{j k}\left(t_{l}, t_{r}\right) \rho_{s s} Q_{j k}^{\dagger}\left(t_{l}, t_{r}\right)\right\}
\end{aligned}
$$

where we have for joint-particle measurements that $P^{j k}$ is a projector $P$ on the $s s$ part and else the unity operator. Since we can construct again commuting operators we can apply also in this case the contextuality criteria revealing the contextual nature even for two different times (case (i) (a) of Fig. 1). On the other hand, if we perform single-particle measurements, the compatibility of the operators cannot be obtained, consequently we cannot apply the criteria. One may think that increasing the number of particles (like in Ref. 14 ) or increasing the number of observables (like in Ref. 19]) may help, however, it is principally not possible to restore the compatibility. A context can only be generated for single particle measurements if Bell's locality assumption in space-time is taken in consideration.

Connection to Bell's theorem. - As is well known if one reduces the number of measurements for the KCBS or Mermin-Peres contextuality test and assumes a tensor product structure of the involved observables, one obtains the Bell-CHSH inequality [18]. The crucial point here is that by Bell's locality assumption one implies indirectly individual particles propagating in spacetime. Still there are the two options of measurements. For joint-particle measurements on a bipartite system we obtain

$$
-2 \operatorname{Tr} \rho_{s s}(t) \leq \operatorname{Tr} \widehat{\operatorname{Bell}} \rho_{s s}(t) \leq 2 \operatorname{Tr} \rho_{s s}(t),
$$

which is violated for all times for any initial state that violates the Bell inequality since $\rho_{s s}(t) / \operatorname{Tr} \rho_{s s}(t)$ is a normalized state. This inequality is a contextuality proof, but no test for Bell's locality hypothesis since both particles are measurement jointly.

Bell's locality hypothesis requires individual particles located at different locations in space-time imposing single-particle measurements and single-particle time evolution. In this case the single-particle measurements do depend on the time choices of $t_{l}, t_{r}$ and the projections, i.e. the operators under investigation become time dependent

$$
\begin{aligned}
\tilde{O}= & \sum_{j, k=s}^{d} 2 K_{j}^{\dagger}\left(t_{l}\right)\left(P^{l}\right)^{j} K_{j}\left(t_{l}\right) \otimes K_{k}^{\dagger}\left(t_{r}\right)\left(P^{r}\right)^{k} K_{k}\left(t_{r}\right) \\
& -K_{j}^{\dagger}\left(t_{l}\right) K_{j}\left(t_{l}\right) \otimes K_{k}^{\dagger}\left(t_{r}\right) K_{k}\left(t_{r}\right)
\end{aligned}
$$

with

$$
(P)^{j}=\left\{\begin{array}{l}
j=s: P \\
j=d: \mathbb{1}
\end{array}\right.
$$

These operators are always commuting (compatible) since they have the natural context of being measured at different instances in space-time. Note in particular, in the case all operators $P^{l}, P^{r}$ are chosen to be the same, we obtain a nontrivial Bell inequality violated by different time choices (distances from the source), exhibiting a kind of "dynamical" nonlocality. Such a type of Bell's inequality being experimentally feasible with a further trick was introduced for entangled decaying $\mathrm{K}$-mesons in Ref. [20].

Conclusions. - The contextual property is conjectured to be key ingredient of quantum theory. We discuss how this property can be revealed in decaying quantum systems under the assumption that the entire time evolution including the decay property is independent of the measurement choices. We found that any criterion based on joint-particle measurements and joint-particle or singleparticle time evolution can be rewritten to display the contextual nature, in principle at any instant in time. 
That proves that the decay property per se is not sensitive to the notion of measurement contextuality.

Interestingly, we find that the standard contextuality criteria can not be applied when we assume singleparticle measurements, because the compatibility requirement is not fulfilled. The requirement can be restored by generating the context via assumption of spacetime-localization leading to state-dependent and decay property dependent Bell-like inequalities.

This findings prove the crucial difference between assigning hidden parameters to measurement outcomes and local hidden parameters to the involved state; such as that the context is achieved by different requirements on the setup: compatible joint-particle measurements or localization assumption in space-time. It illustrates the foundational different concepts of time in time evolutions of states and in space-time with respect to compatible measurement setups.

Acknowledgement: B.C. Hiesmayr acknowledges gratefully the Austrian Science Fund (FWF-P26783). Both authors want to thank the COST-action MP1006 "Fundamental Problems in Quantum Physics" that initiated this work.

* Electronic address: Beatrix.Hiesmayr@univie.ac.at

† Electronic address: jan-ake.larsson@liu.se

[1] E.P. Specker, Dialectica 14, 239 (1960).
[2] S. Kochen and E.P. Specker, Journal of Mathematics and Mechanics 17, 59 (1967).

[3] J.-Å. Larsson, in AIP conference proceedings, Vol. 1424, pp. 211-220 (2012).

[4] R. Raussendorf, Phys. Rev. A 88, 022322 (2013).

[5] M. Howard, J. J. Wallman, V. Veitch and J. Emerson, Nature 510, 351 (2014).

[6] A.A. Klyachko, M.A. Can, S. Binicioğlu and A.S. Shumovsky, Phys. Rev. Lett. 101, 020403 (2008).

[7] A. Peres, J. Phys. A: Math. Gen. 24, L175 (1991).

[8] N. D. Mermin, Physics Today 43, 9 (1990).

[9] J. Bernabeu, N. E. Mavromatos, and P. Villanueva-Perez, Phys. Lett. B 724, 269 (2013).

[10] K. A. Smolinski, Open Syst. Inf. Dyn. 21, 1450003 (2014).

[11] R.A. Bertlmann, W. Grimus and B.C. Hiesmayr, Phys. Rev. A 73, 054101 (2006).

[12] G. Lindblad, Comm. Math. Phys. 48, 119 (1976).

[13] V. Gorini, A. Kossakowski, and E.C.G. Sudarshan, J. Math. Phys. 17, 821 (1976).

[14] N. D. Mermin, Phys. Rev. Lett. 65, 3373 (1990).

[15] E. Gabathulera and P. Pavlopoulos, Physics Reports 403, 303 (2004).

[16] Amelino-Camelia G et.al., Physics with the KLOE-2 experiment at the upgraded DAPHNE European Physical Journal C 68, Number 3, 619 (2010).

[17] B.C. Hiesmayr, Nature: Sci.Rep. 5, 11591 (2015).

[18] J.F. Clauser, M.A. Horne, A. Shimony, R.A. Holt, Phys. Rev. Lett. 23, 880 (1969).

[19] A. Cabello, Phys. Rev. Lett. 101, 210401 (2008).

[20] B.C. Hiesmayr, A. Di Domenico, C. Curceanu, A. Gabriel, M. Huber, J.-A. Larsson, and P. Moskal, Eur. Phys. J. C 72, 1856 (2012). 